\title{
SOME COMMENTS ON METHODOLOGY OF CUBIC RANK TRANSMUTED DISTRIBUTIONS
}

\author{
Monireh HAMELDARBANDI ${ }^{1}$ and Mehmet YILMAZ \\ ${ }^{1}$ Ankara University, Graduate School of Natural and Applied Sciences, 06110, Diskapi, Ankara, \\ TURKEY \\ ${ }^{2}$ Ankara University, Faculty of Science, Department of Statistics, Ankara, TURKEY
}

\begin{abstract}
In this study, at first a new polynomial rank transmutation is proposed. Then, a new cubic rank transmutation is introduced by simplifying the set of transmutation parameters in order to improve its usefulness in statistical modeling. The purpose of this comment is to clarify some issues that exist in the methodology of obtaining the distribution by the cubic transmutation and the stage of proofing it. In this way, both the parameter space is expanded and the process of establishing the cubic transformed distribution family is given.
\end{abstract}

\section{INTRODUCTION}

In this study, we inspire the quadratic rank transmutation map (QRTM) proposed by [16]. The mapping is given as

$$
u \rightarrow u+\lambda u(1-u)
$$

where $u \in[0,1]$ and $\lambda \in[-1,1]$. Using this transmutation many distributions have been derived and still continue to be derived. Beside this, there are also some studies on the modifications of the QRTM. Some of the pioneering works on proposing modified QRTM can be given as follows: 1] proposed a new Weibull distribution by using exponentiated QRTM. 2 generated a new distribution family by considering exponentiated distribution as the baseline distribution. 11] studied a new distribution by taking the baseline distribution as exponentiated exponential distribution. 5 introduced transmuted exponentiated modified Weibull distribution, and $\sqrt{3}$ introduced transmuted exponentiated Lomax distribution. The last three

2020 Mathematics Subject Classification. Primary 05C38, 15A15; Secondary 05A15, 15A18.

Keywords and phrases. Polynomial rank transmutation, cubic rank transmutation, quadratic rank transmutation, transmuted distribution family, order statistics, failure distribution.

monir6685@gmail.com; mehmetyilmaz@ankara.edu.tr-Corresponding author

(D) 0000-0002-3543-3709; 0000-0002-9762-6688. 
studies can be seen as a special case of [2] [9] introduced a new transmutation map by adding extra two parameters to get more flexible distribution. Then, 10 introduced a new Lindley distribution by using this new transmutation map approach. 4] introduced a kind of generalization of QRTM by considering sum of $\mathrm{k}$ - dimensional vector of transmutation parameters. There are two similar studies which are the generalized transmuted $\mathrm{G}$ family by 12 and generalized transmuted Weibull distribution by 13. Also, by taking into account recent works, 15 introduced a new distribution named as transmuted generalized Gamma distribution. They use QRTM to generate this distribution family.

In this study, a new polynomial rank transmutation is proposed additionally to 17. Since the parameter set is still complex, a new cubic rank transmutation is introduced in the light of the idea behind QRTM. In our study, since an extra transmutation parameter is added, the distribution has become more flexible.

\section{Motivation}

17] proposed polynomial rank transmutation map to demonstrate Skew-kurtotic transmutations. Figure 3 of 17 indicates that admissible parameter region. However this region is quite complex structure, the points on Figure 5 of them show some special cases related to family of order statistics up to 3 -sized sample. Under the leadership of this idea, we propose a new polynomial rank transmutation to get simpler structure of parameter region. Let $G(u)$ stand for the polynomial rank transmutation defined on $[0,1]$. Then, we have

$$
G(u)=u+\lambda_{1} u(1-u)+\lambda_{2} u^{2}(1-u)
$$

with $G(0)=0$ and $G(1)=1$. Note that, $\lambda_{1}$ and $\lambda_{2}$ are the transmutation parameters. Parameter region will be defined with following discussion. Since $G$ should be non-decreasing, non-negativity of the first derivative of $G$ with respect to $u$ is examined. Thus, the shape of the parameter region is determined. By calling this derivative with $g$, we have

$$
g\left(u, \lambda_{1}, \lambda_{2}\right)=-3 \lambda_{2} u^{2}-2 u\left(\lambda_{1}-\lambda_{2}\right)+\left(1+\lambda_{1}\right) .
$$

Non-negativity of $g\left(u, \lambda_{1}, \lambda_{2}\right)$ at the end-points, namely the inequalities $g\left(0, \lambda_{1}, \lambda_{2}\right)=$ $1+\lambda_{1} \geq 0$ and $g\left(1, \lambda_{1}, \lambda_{2}\right)=1-\lambda_{1}-\lambda_{2} \geq 0$ both requires that

$$
\begin{aligned}
\lambda_{1} & \geq-1 \\
\lambda_{1}+\lambda_{2} & \leq 1 .
\end{aligned}
$$

From these two inequalities, it is clear that $\lambda_{2} \leq 2$. When the eq. (3) is taken into account, $g\left(u, \lambda_{1}, \lambda_{2}\right)$ is a concave function for $\lambda_{2} \in(0,2]$. As long as the inequality (4) is valid, $g\left(u, \lambda_{1}, \lambda_{2}\right)$ will take non-negative values. For $\lambda_{2} \leq 0$, we will investigate the sufficient conditions on non-negativity of $g\left(u, \lambda_{1}, \lambda_{2}\right)$. In this case, $g\left(u, \lambda_{1}, \lambda_{2}\right)$ has a minimum point since it is a convex function. If this minimum point is within $(0,1)$, the value at that point of the function $g\left(u, \lambda_{1}, \lambda_{2}\right)$ must be 
positive. Accordingly, the minimum point is obtained by taking the derivative of the eq. (3) and equating them to zero as follows:

$$
g^{\prime}\left(u, \lambda_{1}, \lambda_{2}\right)=-6 \lambda_{2} u-2\left(\lambda_{1}-\lambda_{2}\right)=0 \Rightarrow u^{*}=\frac{-\left(\lambda_{1}-\lambda_{2}\right)}{3 \lambda_{2}}
$$

Then, the value of $g\left(u, \lambda_{1}, \lambda_{2}\right)$ at $u^{*}$ must satisfy

$$
g\left(\frac{-\left(\lambda_{1}-\lambda_{2}\right)}{3 \lambda_{2}}, \lambda_{1}, \lambda_{2}\right)=\frac{\lambda_{1}^{2}+\lambda_{1} \lambda_{2}+\lambda_{2}\left(3+\lambda_{2}\right)}{3 \lambda_{2}} \geq 0
$$

Hence, it is necessary to say that the value of the numerator in (6) is non-positive. If this statement given by the numerator is considered as a second order polynomial of $\lambda_{1}$, the roots are given by

$$
\lambda_{1_{1,2}}=\frac{-\lambda_{2} \pm \sqrt{-3 \lambda_{2}\left(\lambda_{2}+4\right)}}{2}
$$

Here, we can say that the condition $-4 \leq \lambda_{2}$ must also occur in order for the roots to be real valued. Thus, under the condition $-4 \leq \lambda_{2}<0$, we have bounds for $\lambda_{1}$ as follows:

$$
\frac{-\lambda_{2}-\sqrt{-3 \lambda_{2}\left(\lambda_{2}+4\right)}}{2} \leq \lambda_{1} \leq \frac{-\lambda_{2}+\sqrt{-3 \lambda_{2}\left(\lambda_{2}+4\right)}}{2}
$$

For these bounds, the numerator in (6) has a negative sign. This leads to the following conclusion: The range of $\lambda_{1}$ is as in (8) for $\lambda_{2} \in[-4,0)$. However, the minimum value of the lower bound in (8) can be -1 , while the maximum value of the upper bound can be 3 . From this we can say that the range of $\lambda_{1}$ is $[-1,3]$. Thus, combining this results, the parameter region for $\left(\lambda_{1}, \lambda_{2}\right)$ appears as shown in the Figure 1

By considering this parameter set of $\left(\lambda_{1}, \lambda_{2}\right)$, many well defined distributions are generated from the eq. (2) with the baseline distribution $F$. Now, let's get a map of the integer values of the pair $\left(\lambda_{2}, \lambda_{1}\right)$ to see the known distributions tabulated in Table 1 .

The distributions specified by the star in Table 1 are described below how they correspond to some known failure distributions.

Let $X_{r: n}$ be the $r$ th order statistic in a sample of size $n$. By noting that, for $\lambda_{1}=-1, \lambda_{2}=-1$ generated distribution indicates the failure distribution of the lifetime of three-component parallel system, namely, this distribution indicates the distribution of the random variable $X_{3: 3}=\max \left\{X_{1}, X_{2}, X_{3}\right\}$ where $X_{1}, X_{2}$ and $X_{3}$ are independent and identically distributed as $F$. Similarly, for $\lambda_{1}=2, \lambda_{2}=-1$ generated distribution indicates the distribution of the random variable $X_{1: 3}=$ $\min \left\{X_{1}, X_{2}, X_{3}\right\}$. For $\lambda_{1}=-1, \lambda_{2}=1$ generated distribution indicates the distribution of $\max \left\{X_{1}, \min \left\{X_{2}, X_{3}\right\}\right\}$. For $\lambda_{1}=0, \lambda_{2}=1$ generated distribution indicates the distribution of $\min \left\{X_{1}, \max \left\{X_{2}, X_{3}\right\}\right\}$. For $\lambda_{1}=-1, \lambda_{2}=2$ generated distribution indicates the failure distribution of the lifetime of the three-outof- two system, namely, this distribution indicates the distribution of the random 


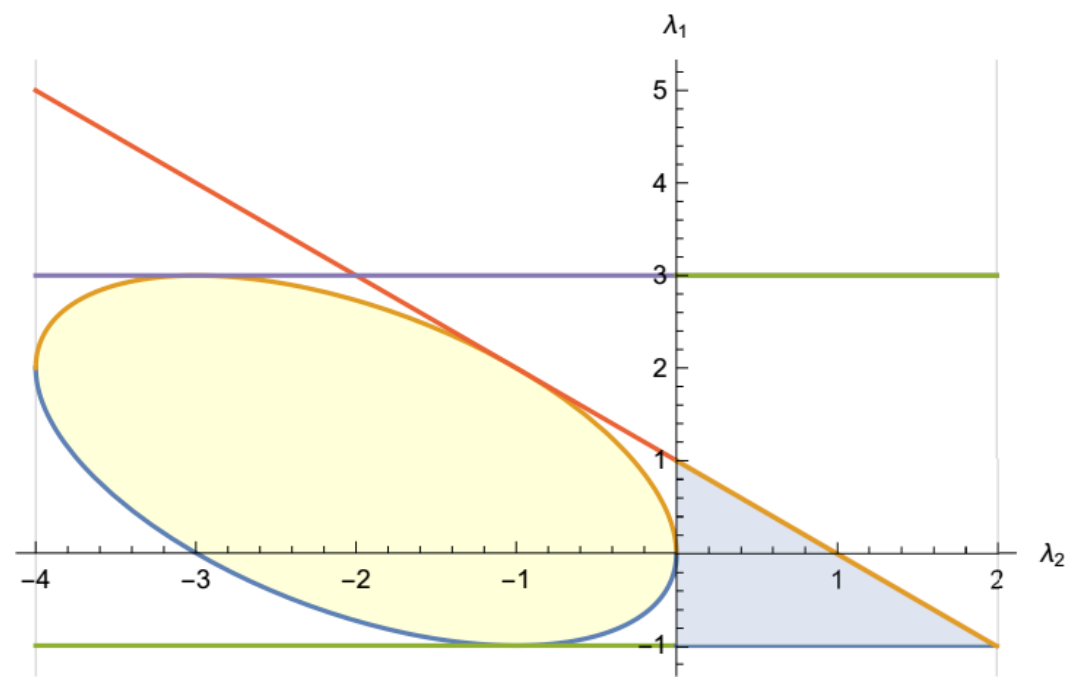

FiguRE 1. Valid parameter set (ellipsoid and triangle on the rightside )

TABLE 1. Some generated distributions according to special cases for the parameter values

\begin{tabular}{|l|l|l|l|l|l|}
\hline$\lambda_{2}$ & $\lambda_{1}$ & Some Generated Distributions & $\lambda_{2}$ & $\lambda_{1}$ & Some Generated Distributions \\
\hline-4 & 2 & $4 F^{3}-6 F^{2}+3 F$ & -1 & 0 & $F^{3}-F^{2}+F$ \\
\hline-3 & 0 & $3 F^{3}-3 F^{2}+F$ & -1 & 1 & $F^{3}-2 F^{2}+2 F$ \\
\hline-3 & 1 & $3 F^{3}-4 F^{2}+2 F$ & -1 & 2 & $F^{3}-3 F^{2}+3 F^{*}$ \\
\hline-3 & 2 & $3 F^{3}-5 F^{2}+3 F$ & 0 & -1 & $F^{2 *}$ \\
\hline-3 & 3 & $3 F^{3}-6 F^{2}+4 F$ & 0 & 0 & $F^{*}$ \\
\hline-2 & 0 & $2 F^{3}-2 F^{2}+F$ & 0 & 1 & $2 F-F^{2 *}$ \\
\hline-2 & 1 & $2 F^{3}-3 F^{2}+2 F$ & 1 & -1 & $2 F^{2}-F^{3 *}$ \\
\hline-2 & 2 & $2 F^{3}-4 F^{2}+3 F$ & 1 & 0 & $F^{2}+F-F^{3 *}$ \\
\hline-1 & -1 & $F^{3 *}$ & 2 & -1 & $3 F^{2}-2 F^{3 *}$ \\
\hline
\end{tabular}

variable $X_{2: 3}=\max \left\{\min \left\{X_{1}, X_{2}\right\}, \min \left\{X_{1}, X_{3}\right\}, \min \left\{X_{2}, X_{3}\right\}\right\}$. On the other hand, for $\lambda_{1}=-1, \lambda_{2}=0$ generated distribution indicates the failure distribution of the lifetime of the two-component parallel system, namely distribution of $X_{2: 2}=\max \left\{X_{1}, X_{2}\right\}$. For $\lambda_{1}=1, \lambda_{2}=0$ generated distribution indicates the failure distribution of the lifetime of the two-component series system, namely distribution of $X_{1: 2}=\min \left\{X_{1}, X_{2}\right\}$.

In this case, in addition to the known distributions introduced by the quadratic transmutation, more informative distribution functions occure. However, the set of 
the transformation parameters of the proposed cubic transmutation is still complicated.

In order to eliminate of this complexity, by referring to the concept of reliability evaluation of coherent system by using signature (see, 6, 6, 7] ), we come up with an idea inspired by both works of $[16,18]$ as follows:

$$
\operatorname{Pr}\left(X_{2: 2} \leq t\right)=\operatorname{Pr}\left(\max \left\{X_{1}, X_{2}\right\} \leq t\right)=F^{2}(t)
$$

and

$$
\operatorname{Pr}\left(X_{1: 2} \leq t\right)=\operatorname{Pr}\left(\min \left\{X_{1}, X_{2}\right\} \leq t\right)=2 F(t)-F^{2}(t)
$$

where $F(t)$ indicates the failure distribution of the component lifetime, namely, $\operatorname{Pr}\left(X_{1} \leq t\right)=F(t)$. Hence there exists a stochastic ordering relation such as $X_{1: 2} \prec_{s t} X \prec_{s t} X_{2: 2}$. In this case, these three failure distributions can be ordered as $F^{2}(t) \leq F(t) \leq 2 F(t)-F^{2}(t)$. From the latter inequality, we can say that $F(t)$ is represented by a convex combination of $2 F(t)-F^{2}(t)$ and $F^{2}(t)$ where the value of the combination parameter is $\frac{1}{2}$. On the other hand, it is possible to obtain many distributions besides $F$. Let $G$ stand for the distribution obtained by this convex combination. Then, for $\delta \in[0,1]$, we have

$$
\begin{gathered}
G(t)=\delta\left(2 F(t)-F^{2}(t)\right)+(1-\delta)\left(F^{2}(t)\right) \\
=2 \delta F(t)+(1-2 \delta) F^{2}(t)
\end{gathered}
$$

Here, the combination parameter is reparametrized by taking $\delta=\frac{1+\lambda}{2}$ to attain quadratic rank transmutation. Now, the new parameter $\lambda$ takes the values in $[-1,1]$. As can be seen immediately, $\lambda=0$ corresponds to $\delta=\frac{1}{2}$. In eq. (9), substituting $\delta$ by $\lambda$, we have

$$
G(t)=(1+\lambda) F(t)-\lambda F^{2}(t) .
$$

The above expression is the quadratic rank trasmutation proposed by 16 . Now, we concentrate on 3-component systems with similar thinking. Let $X_{1}, X_{2}$ and $X_{3}$ be independent random variables distributed as $F$. Let $X_{r: 3}$ denote $r$ th order statistic of $\left(X_{1}, X_{2}, X_{3}\right)$ with corresponding distribution $F_{r: 3}$. Then we have

$$
\begin{gathered}
F_{3: 3}(t)=\operatorname{Pr}\left(X_{3: 3} \leq t\right)=\operatorname{Pr}\left(\max \left\{X_{1}, X_{2}, X_{3}\right\} \leq t\right)=F^{3}(t) \\
F_{2: 3}(t)=\operatorname{Pr}\left(X_{2: 3} \leq t\right) \\
=\operatorname{Pr}\left(\max \left\{\min \left\{X_{1}, X_{2}\right\}, \min \left\{X_{1}, X_{3}\right\}, \min \left\{X_{2}, X_{3}\right\}\right\} \leq t\right) \\
=3 F^{2}(t)-2 F^{3}(t) \\
F_{1: 3}(t)=\operatorname{Pr}\left(X_{1: 3} \leq t\right)=\operatorname{Pr}\left(\min \left\{X_{1}, X_{2}, X_{3}\right\} \leq t\right) \\
=3 F(t)-3 F^{2}(t)+F^{3}(t) .
\end{gathered}
$$

According to 18, the properties $F_{3: 3} \leq F_{2: 3} \leq F_{1: 3}$ and $F=\frac{1}{3} F_{3: 3}+\frac{1}{3} F_{2: 3}+$ $\frac{1}{3} F_{1: 3}$. are hold. In other words, $F$ can be represented by a convex combination of $F_{1: 3}, F_{2: 3}$ and $F_{3: 3}$. On the other hand, there is also an ordering for $F$ such 
that $F_{3: 3} \leq F \leq F_{1: 3}$. If $F_{2: 3}$ is also included in this ordering, we have for $F \geq \frac{1}{2}$, $F_{3: 3} \leq F \leq F_{2: 3} \leq F_{1: 3}$ and for $F<\frac{1}{2}, F_{3: 3} \leq F_{2: 3} \leq F \leq F_{1: 3}$. Hence, we can suggest a convex combination to cover both ordering situations. Our aim is to determine exactly where $F$ is. In this case, we can write the following convex combination obtained by $F_{1: 3}$ and $F_{2: 3}$, called as $G^{*}$.

$$
G^{*}=\delta_{1} F_{1: 3}+\left(1-\delta_{1}\right) F_{2: 3}
$$

where $\delta_{1} \in[0,1]$. Now, let's write a convex combination between $G^{*}$ and $F_{3: 3}$. Denoting this convex combination by $G$, we have

$$
G=\delta_{2} G^{*}+\left(1-\delta_{2}\right) F_{3: 3}
$$

where $\delta_{2} \in[0,1]$. Combining with the equations (14) and (15), we obtain $G$ as

$$
G=\delta_{2} \delta_{1} F_{1: 3}+\delta_{2}\left(1-\delta_{1}\right) F_{2: 3}+\left(1-\delta_{2}\right) F_{3: 3} .
$$

If the notation $F$ is used for the representation of $F_{r: 3}$, and rearranging with respect to polynomial degree of $F$, the following expression is obtained:

$$
\begin{aligned}
G & =\delta_{2} \delta_{1}\left(3 F-3 F^{2}+F^{3}\right)+\delta_{2}\left(1-\delta_{1}\right)\left(3 F^{2}-2 F^{3}\right)+\left(1-\delta_{2}\right) F^{3} \\
& =3 \delta_{1} \delta_{2} F+3 \delta_{2}\left(1-2 \delta_{1}\right) F^{2}+\left(1-3 \delta_{2}+3 \delta_{1} \delta_{2}\right) F^{3}
\end{aligned}
$$

Undoubtedly, $G$ is a distribution function. However, reparameterization is made on the model in order to achieve the similar structure of the quadratic rank transmutation. Now, by taking $w_{1}=\delta_{1} \delta_{2}$ and $w_{2}=\delta_{2}-\delta_{1} \delta_{2}$, eq. (17) can be rewritten as follows:

$$
G=3 w_{1} F+3\left(w_{2}-w_{1}\right) F^{2}+\left(1-3 w_{2}\right) F^{3}
$$

where $w_{1}, w_{2} \in[0,1]$. In eq. 18, , by the reparametrizating as $w_{1}=\frac{1+\lambda_{1}}{3}$ and $w_{2}=\frac{1+\lambda_{2}}{3}$, we have

$$
G=\left(1+\lambda_{1}\right) F+\left(\lambda_{2}-\lambda_{1}\right) F^{2}-\lambda_{2} F^{3}
$$

where $\lambda_{1}, \lambda_{2} \in[-1,2]$. Since $\delta_{2}=w_{1}+w_{2}$, the parameter set is also constrained by the condition $\lambda_{1}+\lambda_{2} \leq 1$. Consequently, the parameter set of $\lambda_{1}$ and $\lambda_{2}$ is presented in a simpler form than the parameter region given in Figure 1. This transmutation defined in eq. (19) is called as cubic rank transmutation and transformed distribution $G$ is named as CRT-F.

As can be seen immediately, CRT-F defines a quadratic rank transmuted distribution at $\lambda_{2}=0$, and $\lambda_{1}=\lambda_{2}=0$ gives the baseline distribution $F$. For this reason, CRT-F can be seen as a generalized form of QRT. The parameter set of $\lambda_{1}$ and $\lambda_{2}$, which is defined as $\left\{\left(\lambda_{1}, \lambda_{2}\right): \lambda_{1}, \lambda_{2} \in[-1,2], \lambda_{1}+\lambda_{2} \leq 1\right\}$ can be figure out in Figure 2 .Now, referring to the integer values of $\lambda_{1}$ and $\lambda_{2}$, we can determine the generated distribution functions by the Table 2 .

Identifications given in Table 2 show that Table 1 of 17 is included by CRT-F according to special choices of transmutation parameters. 


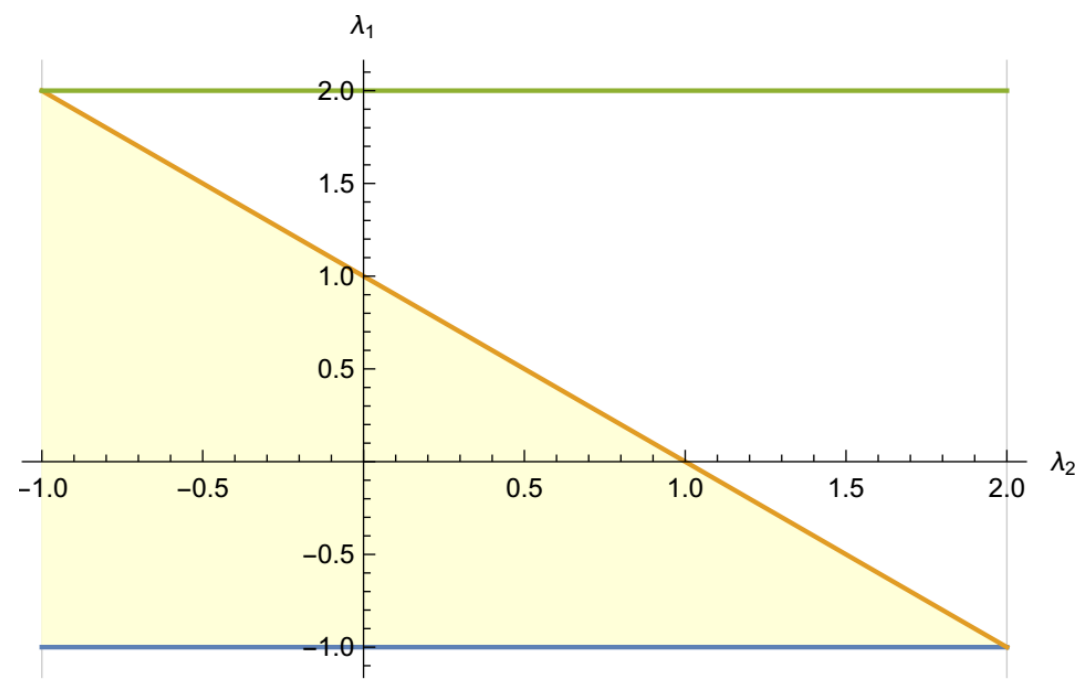

FiguRE 2. Region of the parameter set of CRT-F

TABLE 2. Identifications of CRT-F distribution for special values of transmutation parameters

\begin{tabular}{|l|l|l|l|}
\hline$\lambda_{1}$ & $\lambda_{2}$ & CRT-F & Identification \\
\hline-1 & -1 & $F^{3}$ & Distribution of $T_{3: 3}$ \\
\hline-1 & 0 & $F^{2}$ & Distribution of $T_{2: 2}$ \\
\hline-1 & 1 & $2 F^{2}-F^{3}$ & Distribution of $\max \left\{X_{1}, \min \left\{X_{2}, X_{3}\right\}\right\}$ \\
\hline-1 & 2 & $3 F^{2}-2 F^{3}$ & Distribution of $T_{2: 3}$ \\
\hline 0 & -1 & $F^{3}-F^{2}+F$ & $\frac{1}{3} F_{1: 3}+\frac{2}{3} F_{3: 3}$ \\
\hline 0 & 0 & $F$ & Baseline Distribution \\
\hline 0 & 1 & $F^{2}+F-F^{3}$ & Distribution of $\min \left\{X_{1}, \max \left\{X_{2}, X_{3}\right\}\right\}$ \\
\hline 1 & -1 & $F^{3}-2 F^{2}+2 F$ & $\frac{2}{3} F_{1: 3}+\frac{1}{3} F_{3: 3}$ \\
\hline 1 & 0 & $2 F-F^{2}$ & Distribution of $T_{1: 2}$ \\
\hline 2 & -1 & $F^{3}-3 F^{2}+3 F$ & Distribution of $T_{1: 3}$ \\
\hline
\end{tabular}

Note that, by taking into account the parameter set of $(19)$, the distribution family CRT-F is different as compared with the families proposed by 8,14 .

8 proposed a cubic rank transmuted distribution family motivated by a study of [17. The paper contained one theorem (referred to as Theorem 2.1), deriving cubic transmuted distribution. Here, We would like to point out that the result of Theorem 2.1 can be reduced to an explicit and understandable form. 
Parameter space of eq. (3) of Theorem 2.1 given by 8 needs to be revised according to mixture probabilities $\pi_{i}(i=1,2,3)$. Otherwise, eq. (3) does not provide the distribution function in some cases of $\lambda_{1}$ and $\lambda_{2}$. For instance, suppose that $\lambda_{1}=0$ and $\lambda_{2}=-1$ in eq. (3), then we have

$$
F(x)=-G^{2}(x)+2 G^{3}(x)=G^{2}(x)(2 G(x)-1) .
$$

Thus, the function $F(x)$ is positively signed when $x$ is greater than $G^{-1}(1 / 2)$ which is the median of $G(x)$. Otherwise, $F(x)$ is negatively signed. More generally, according to eq. (3), we have the second-order convex polynomial as $\varphi(u)=$ $\lambda_{1}+\left(\lambda_{2}-\lambda_{1}\right) u+\left(1-\lambda_{2}\right) u^{2}$. After some algebraic manipulation, we say that having positively sign of $\varphi(u)$ depends on $\lambda_{1} \geq \frac{\left(\lambda_{1}+\lambda_{2}\right)^{2}}{4}$. Furthermore, in proof of Theorem 2.1, one condition is missing which comes with $0 \leq \pi_{1}+\pi_{2} \leq 1$. To clarify the above claims, recall the distribution of order statistics associated with sample size of 3 :

$$
\begin{gathered}
\operatorname{Pr}\left(X_{1: 3} \leq x\right)=1-\operatorname{Pr}\left(X_{1: 3}>x\right)=1-(1-F(x))^{3}=3 F(x)-3 F^{2}(x)+F^{3}(x), \\
\operatorname{Pr}\left(X_{2: 3} \leq x\right)=3 F(x)^{2}-2 F^{3}(x), \\
\operatorname{Pr}\left(X_{3: 3} \leq x\right)=F^{3}(x) .
\end{gathered}
$$

Now, a new random variable $T$ is defined by mixing the above order statistics as follows:

$$
T= \begin{cases}X_{1: 3}, & \text { with probability } \pi_{1} \\ X_{2: 3}, & \text { with probability } \pi_{2} \\ X_{3: 3}, & \text { with probability } \pi_{3},\end{cases}
$$

where $\pi_{1}+\pi_{2}+\pi_{3}=1$. Then the distribution of $T$ is as follows:

$$
\begin{aligned}
\operatorname{Pr}(T \leq t) & =\pi_{1}\left(3 F(t)-3 F^{2}(t)+F^{3}(t)\right)+\pi_{2}\left(3 F^{2}(t)-2 F^{3}(t)\right)+\pi_{3}\left(F^{3}(t)\right) \\
& =\left(3 \pi_{1}\right) F(t)+3\left(\pi_{2}-\pi_{1}\right) F^{2}(t)+\left(\pi_{1}-2 \pi_{2}+\pi_{3}\right) F^{3}(t) .
\end{aligned}
$$

By letting $\pi_{3}=1-\pi_{1}-\pi_{2}$ then we have

$$
\operatorname{Pr}(T \leq t)=3 \pi_{1} F(t)+3\left(\pi_{2}-\pi_{1}\right) F^{2}(t)+\left(1-3 \pi_{2}\right) F^{3}(t) .
$$

Since $\pi_{i} \in[0,1],(i=1,2,3)$, appropriate parameterization for $\pi_{1}$ and $\pi_{2}$, can be taken into account as $3 \pi_{1}=1+\lambda_{1}$, and $3 \pi_{2}=1+\lambda_{2}$. New parameters are both in the interval $[-1,2]$. Recalling the condition $0 \leq \pi_{1}+\pi_{2} \leq 1$, hence we have $-2 \leq \lambda_{1}+\lambda_{2} \leq 1$. Accordingly, latter probability is as follows:

$$
\operatorname{Pr}(T \leq t)=\left(1+\lambda_{1}\right) F(t)+\left(\lambda_{2}-\lambda_{1}\right) F^{2}(t)-\lambda_{2} F^{3}(t),
$$

with $\lambda_{1}, \lambda_{2} \in[-1,2]$ and $-2 \leq \lambda_{1}+\lambda_{2} \leq 1$. It should be noted that this is also obtained by 19 .

Therefore, based on the assumptions in proof of Theorem 2.1., eq. (3) cannot be obtained. Also, comparing with the family introduced by [14, they give the similar cubic transmuted distribution family with a narrower parameter space as $\lambda_{1}, \lambda_{2} \in[-1,1]$ and $-2 \leq \lambda_{1}+\lambda_{2} \leq 1$. 


\section{Conclusion}

In this article, we propose a new version of polynomial rank transmutation. Since the parameter set is still complex, a new cubic rank transmutation is introduced in the light of the idea behind QRTM technique. Compared to the two techniques in the literature, it is seen that the proposed technique covers them in terms of parameter space.

\section{REFERENCES}

[1] Abd El Hady, N. E., Exponentiated transmuted weibull distribution a generalization of the weibull distribution, International Journal of Mathematical, Computational, Physical, Electrical and Computer Engineering, 8 (6) (2014), 903 - 911.

[2] Alizadeh, M., Merovci, F., Hamedani, G. G., Generalized transmuted family of distributions: properties and applications, Hacettepe Journal of Mathematics and Statistics, 46 (2017), 645-667.

[3] Ashour, S. K., Eltehiwy, M. A., Transmuted exponentiated lomax distribution, Aust J Basic Appl Sci, 7 (7) (2013), 658-667.

[4] Das, K. K., Barman, L., On some generalized transmuted distributions, Int. J. Sci. Eng. Res, 6 (2015), 1686-1691.

[5] Eltehiwy, M., Ashour, S., Transmuted exponentiated modified weibull distribution, International Journal of Basic and Applied Sciences, 2 (3) (2013), 258-269.

[6] Eryilmaz, S., Computing reliability indices of repairable systems via signature, Journal of Computational and Applied Mathematics, 260 (2014), 229 - 235, https://dx.doi.org/https://doi.org/10.1016/j.cam.2013.09.023.

[7] Franko, C., Ozkut, M., Kan, C., Reliability of coherent systems with a single cold standby component, Journal of Computational and Applied Mathematics, 281 (2015), 230 - 238, https://dx.doi.org/https://doi.org/10.1016/j.cam.2014.12.029.

[8] Granzotto, D., Louzada, F., Balakrishnan, N., Cubic rank transmuted distributions: inferential issues and applications, Journal of Statistical Computation and Simulation, 87 (14) (2017), 2760-2778.

[9] Mansour, M. M., Enayat, M. A., Hamed, S. M., Mohamed, M. S., A new transmuted additive weibull distribution based on a new method for adding a parameter to a family of distributions, Int. J. Appl. Math. Sci, 8 (2015), 31-51.

[10] Mansour, M. M., Mohamed, S. M., A new generalized of transmuted lindley distribution, Appl. Math. Sci, 9 (2015), 2729-2748.

[11] Merovci, F., Transmuted exponentiated exponential distribution, Mathematical Sciences and Applications E-Notes, 1 (2) (2013), 112-122.

[12] Nofal, Z. M., Afify, A. Z., Yousof, H. M., Cordeiro, G. M., The generalized transmuted-g family of distributions, Communications in Statistics-Theory and Methods, 46 (8) (2017), $4119-4136$.

[13] Nofal, Z. M., El Gebaly, Y. M., The generalized transmuted weibull distribution for lifetime data, Pakistan Journal of Statistics and Operation Research, 13 (2) (2017), 355-378.

[14] Rahman, M. M., Al-Zahrani, B., Shahbaz, M. Q., A general transmuted family of distributions, Pakistan Journal of Statistics and Operation Research, 14 (2) (2018).

[15] Saboor, A., Khan, M. N., Cordeiro, G. M., Pascoa, M. A., Ramos, P. L., Kamal, M., Some new results for the transmuted generalized gamma distribution, Journal of Computational and Applied Mathematics, 352 (2019), 165 - 180, https://dx.doi.org/https://doi.org/10.1016/j.cam.2018.12.002. 
[16] Shaw, W. T., Buckley, I. R. C., The alchemy of probability distributions: Beyond gramcharlier \& cornish-fisher expansions, and skew-normal or kurtotic-normal distributions, Submitted, Feb, 7 (2007), 64.

[17] Shaw, W. T., Buckley, I. R. C., The alchemy of probability distributions: beyond GramCharlier expansions, and a skew-kurtotic-normal distribution from a rank transmutation map, ArXiv e-prints (Jan. 2009).

[18] Yilmaz, M., Potas, N., Topcu, B., Reliability properties of systems with three dependent components, In Chaos, Complexity and Leadership 2013, Springer, 2015, pp. 117-127. 